\title{
Painéis compensados sarrafeados produzidos com Pinus oocarpa, Castilla ulei e Acrocarpus fraxinifolius
}

\author{
Eduardo Pinati ${ }^{1}$, Douglas Lamounier Faria ${ }^{1}$, Rafael Farinassi Mendes ${ }^{1}$, Lourival Marin Mendes ${ }^{1}$, \\ Thiago de Paula Protásio², José Benedito Guimarães Júnior ${ }^{1 \star}$
}

\footnotetext{
${ }^{1}$ Departamento de Ciências Florestais, Universidade Federal de Lavras, Lavras, Minas Gerais, Brasil.

${ }^{2}$ Curso de Engenharia Florestal, Universidade Federal Rural da Amazônia, Parauapebas, Pará, Brasil.
}

\begin{abstract}
RESUMO O objetivo deste trabalho foi estudar as propriedades físicas e mecânicas de compensados sarrafeados produzidos a partir de resíduos das madeiras de Pinus oocarpa, Castilla ulei e Acrocarpus fraxinifolius. Essas espécies constituíram o miolo dos painéis, enquanto as capas foram produzidas com lâminas de Pinus oocarpa. Foram analisadas as seguintes propriedades das madeiras: densidades básica e aparente, retratibilidade, umidade, módulos de elasticidade e ruptura no ensaio de flexão estática. O adesivo PVA foi utilizado na colagem dos sarrafos e das lâminas. Utilizou-se a gramatura de $160 \mathrm{~g} / \mathrm{m}^{2} \mathrm{entre}$ os sarrafos e $320 \mathrm{~g} / \mathrm{m}^{2}$ na colagem das lâminas. Os painéis foram produzidos com dimensões de 300x300x28mm. Foram avaliadas as seguintes propriedades: umidade; densidade aparente; absorção de água; cisalhamento na linha de cola e os módulos de elasticidade e ruptura no ensaio de flexão estática. Os resultados para as propriedades físicas das madeiras mostraram que não houve diferença significativa entre as espécies. Já para as propriedades avaliadas para os painéis compensados sarrafeados, a madeira de Pinus oocarpa presente na capa e miolo proporcionou o maior valor médio para módulo de ruptura e módulo de elasticidade, paralelo e perpendicular.
\end{abstract}

Palavras-chave: Resíduo; propriedades físicas e mecânicas; PVA.

\section{Blockboard plywood produced from Pinus oocarpa, Castilla ulei and Acrocarpus fraxinifolius}

\begin{abstract}
The objective of this work was to study the physical and mechanical properties of plywood panels produced from wood residues of Pinus oocarpa, Castilla ulei and Acrocarpus fraxinifolius. These species constituted the core of the panels, while the layers were produced with slides of Pinus oocarpa. The following properties of the woods were analyzed: basic and apparent densities, retractability, moisture and mechanical characterization. The PVA adhesive was used in the bonding of slats and slides. The weight of $160 \mathrm{~g} / \mathrm{m}^{2}$ was used between slats and $320 \mathrm{~g} / \mathrm{m}^{2}$ in the bonding of the slides. The panels were produced with dimensions of 300x300x $28 \mathrm{~mm}$. The following properties were evaluated: moisture; apparently density; water absorption; shear in the glue line and the modulus of elasticity and rupture in the static bending test. The results for the physical properties of the woods showed that there was no significant difference between the species. As for the properties evaluated for the composite panels, the Pinus oocarpa wood present in the cover and core gave the highest average value for modulus of rupture and modulus of elasticity, parallel and perpendicular.
\end{abstract}

Keywords: Wastes; physical and mechanical properties; PVA.

\section{Introdução}

No Brasil, a geração de resíduos florestais é muito variável, com estimativa de 30 milhões de toneladas de resíduos de madeira no ano de 2008 e 87,8 milhões em 2020
(MINISTÉRIO DO MEIO AMBIENTE - MMA, 2009; BORTOLIN et al., 2012). Para a STCP (2011) são geradas entre 75 e $90 \%$ de resíduos em todo o processo produtivo florestal e madeireiro, considerando desde a retirada da árvore da floresta até o produto final. 
O aumento significativo na demanda pela madeira de pinus e eucalipto nas últimas duas décadas tem motivado as empresas de base florestal pela ampliação das áreas de plantios para equilibrar a relação oferta-demanda de madeira para fins industriais. Os resíduos de madeira gerados por seu processamento podem deixar de ser risco ao meio ambiente e passar a gerar lucro para a empresa que os produz, criando alternativas para sua utilização como matéria-prima, como por exemplo em painéis de madeira reconstituída (LIMA; SILVA, 2005).

Os materiais residuais provenientes de madeira são uma grande fonte de fibras, que quando processadas adequadamente, podem ser utilizadas para a produção de compensados, painéis colados, componentes de móveis e painéis de revestimento (IWAKIRI, 2005). Um desses painéis é o painel sarrafeado, definido como um compensado formado por um miolo de sarrafos colados lateralmente entre si, com lâminas de madeira coladas perpendicularmente entre si dos dois lados dos sarrafos, sendo duas contracapas e duas capas de cada lado do compensado (IWAKIRI, 2005). A utilização do compensado sarrafeado tem aumentado, devido ao seu menor custo de produção e facilidade no manuseio quando comparado ao compensado multilaminado convencional, além do seu bom rendimento.

Desta forma, o presente trabalho teve como objetivo avaliar as propriedades físicas e mecânicas de compensados sarrafeados produzidos a partir de madeira de Pinus oocarpa, Castilla ulei e Acrocarpus fraxinifolius.

\section{Material e Métodos}

Os resíduos madeireiros utilizados para a produção dos miolos dos compensados foram adquiridos em madeireira na cidade de Lavras - MG. Os sarrafos utilizados no trabalho foram obtidos a partir de tábuas das espécies Pinus oocarpa, Castilla ulei e Acrocarpus fraxinifolius, sendo as espécies mais comercializadas no município. Para a produção das lâminas da capa e contracapa foi realizada a laminação da madeira de Pinus oocarpa. Os sarrafos foram obtidos através do corte de tábuas de dimensões 1200x60x20mm das três espécies.

Após o processamento, os sarrafos foram armazenados, para que as peças atingissem a umidade de equilíbrio higroscópico. Os mesmos foram climatizados à temperatura de $20 \pm 3^{\circ} \mathrm{C}$ e umidade relativa de $65 \pm 5 \%$, estabilizando suas dimensões e massa, facilitando o processo de colagem dos sarrafos.

Para a caracterização física, química e mecânica dos sarrafos foram realizados os seguintes ensaios: umidade, densidade aparente e retratibilidade de acordo com a NBR 7190 (ABNT, 1997), densidade básica de acordo com a NBR 11941 (ABNT, 2003), flexão estática segundo a norma D 143 (ASTM, 1995), análise de extrativos totais de acordo com a norma NBR 14853 (ABNT, 2010), teor de lignina insolúvel de acordo com a norma NBR 7989 (ABNT, 2010), teor de cinzas de acordo com a norma NBR 13999 (ABNT, 2017) e por diferença, o teor de holocelulose.

Foram produzidos painéis compensados nas dimensões de 300x300x25mm, com quatro repetições por tratamento (espécies). Cada sarrafo apresentou dimensões de $300 \times 25 \times 20 \mathrm{~mm}$.

Os sarrafos foram inicialmente colados lado a lado com o uso de adesivo PVA (Poliacetato de Vinila), com gramatura de $160 \mathrm{~g} / \mathrm{m}^{2}$, e para que eles ficassem unidos, foram utilizados grampos do tipo "sargento", que ficavam prensados por um período de 24 horas, conforme mostra a Figura 1a.

Posteriormente, foram coladas as capas e contracapas com o uso de cola branca (PVA) com gramatura de $320 \mathrm{~g} / \mathrm{m}^{2}$. As contracapas foram coladas primeiramente, com as fibras perpendiculares as fibras dos sarrafos, e logo após com suas fibras em sentido perpendicular ao da contracapa, formando o painel (Figura $1 b)$. 


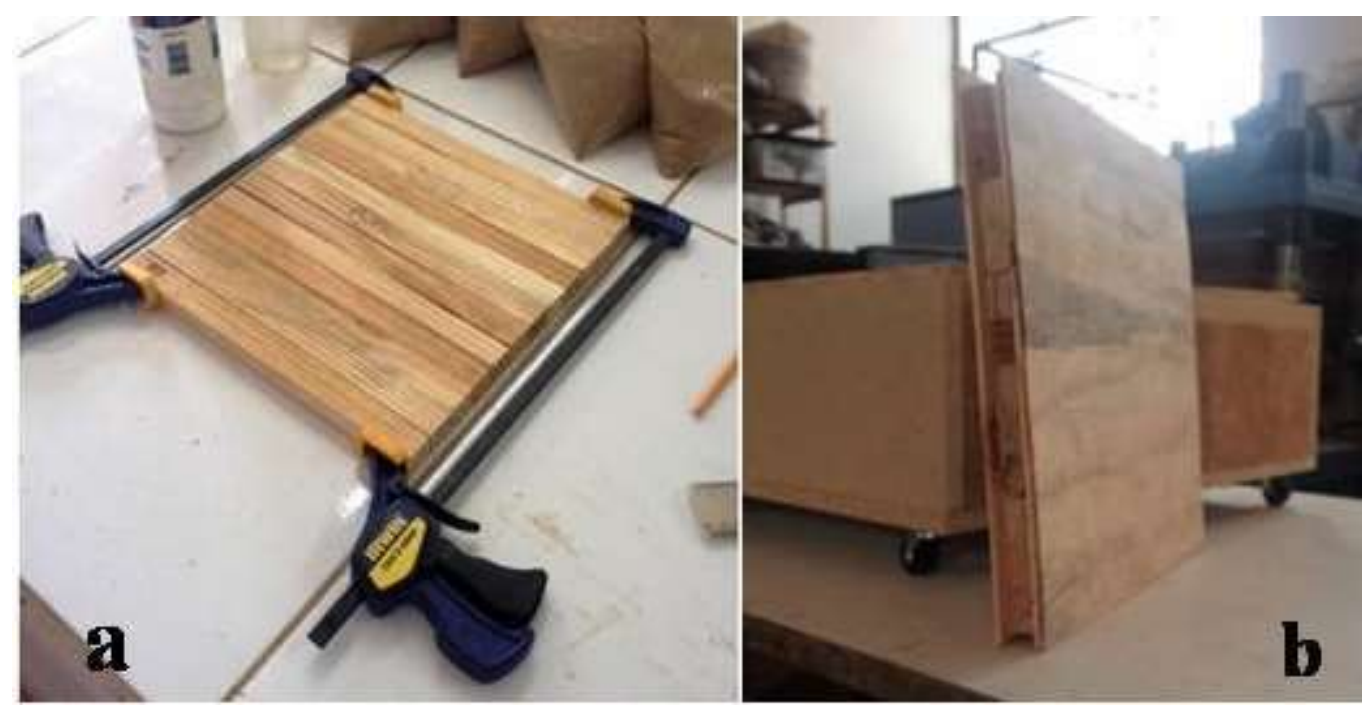

Figura 1 - a) Processo de prensagem dos sarrafos pelos grampos tipo "sargento" pelo período de 24 horas, b) Painel compensado sarrafeado com dimensões de 300x300x25mm.

Figure 1 - a) Process of pressing of the battens by the "sergeant" type clamps for the period of 24 hours, b) Compacted plywood panel with dimensions of 300x300x $25 \mathrm{~mm}$.

A prensagem foi feita a frio com utilização de uma prensa manual, onde os painéis permaneceram por 24 horas a pressão de 1,0 MPa. Posteriormente a prensagem, os painéis foram climatizados a temperatura de $20 \pm 2^{\circ} \mathrm{C}$ e umidade relativa de $65 \pm 3 \%$ até massa constante.

As propriedades avaliadas nos painéis foram: teor de umidade NBR 9484 (ABNT, 2011), absorção de água NBR 9486 (ABNT, 2011), densidade aparente NBR 9485 (ABNT, 2011), cisalhamento na linha de cola NBR ISO 12466-1 (ABNT, 1986) e flexão estática NBR 9533 (ABNT, 2012).

Para comparar os tratamentos como as diferentes espécies foi utilizado o delineamento inteiramente casualizado (DIC). As médias dos tratamentos foram comparadas pelo teste de Tukey em 5\% de significância.

\section{Resultados e Discussão}

\section{Propriedades físicas, químicas e mecânicas das}

\section{madeiras.}

Na Tabela 1 encontram-se os valores médios de umidade na base seca, densidade aparente e densidade básica das três espécies estudadas de madeiras.
Os valores médios para umidade não diferiram estatisticamente entre si, com valores variando entre 10,57 e $10,81 \%$. Este fato ocorreu em consequência do período de climatização adequado, com temperatura e umidade controladas, pelo qual as madeiras passaram. De acordo com Iwakiri (2005), as lâminas para painéis compensados devem estar na faixa de 6 a 12\% de umidade para uma melhor colagem.

Trianoski et al. (2013a) avaliando as propriedades físicas de Acrocarpus fraxinifolius obtiveram valor de $0,45 \mathrm{~g} / \mathrm{cm}^{3}$ para densidade básica; mesmo valor encontrado por Iwakiri et al. (2014). Mattos et al. (2011) obtiveram resultados variando entre 0,35 a $0,45 \mathrm{~g} / \mathrm{cm}^{3}$ para madeiras de Pinus oocarpa, conforme a altura da árvore. Trianoski et al. (2013b) obtiveram valor de $0,54 \mathrm{~g} / \mathrm{cm}^{3}$ para Pinus oocarpa. Para Mendes et al. (1999) a densidade básica é um dos principais parâmetros de qualidade da madeira para utilização como matéria-prima industrial, sendo definida pela relação entre o peso da madeira seca e o seu volume saturado.

As madeiras de Acrocarpus fraxinifolius, Pinus oocarpa e Castilla ulei são consideradas madeiras de média densidade, apresentando valores de densidade aparente entre 0,55 e 0,75 
$\mathrm{g} / \mathrm{cm}^{3}$. A densidade da madeira pode ser considerada como um bom indicador das propriedades de resistência da madeira (BRAZ et al., 2014).

$\mathrm{Na}$ Tabela 2, estão apresentados os valores médios da retratibilidade radial; e tangencial, da variação volumétrica e do coeficiente de anisotropia das três espécies estudadas de madeiras.

Para a contração radial, os valores variaram entre $2,51 \%$ e $5,77 \%$, sendo o menor valor médio apresentado pelo Acrocarpus fraxinifolius e o valor mais elevado, obtido a partir do Castilla ulei. Comparando as espécies utilizadas, é possível constatar que apenas houve diferença estatística significativa para a madeira de Castilla ulei, que obteve o maior valor médio.

Em relação às contrações tangencial e volumétrica, os valores médios variaram de 4,29\% e 9,33\% para contração tangencial e $8,63 \%$ a $17,82 \%$ para contração volumétrica, sendo o Castilla ulei a espécie que apresentou o maior valor médio, o qual diferiu estatisticamente de todas as espécies. $\mathrm{O}$ menor valor foi apresentado pelo Pinus oocarpa, sendo este estatisticamente diferente de todas as demais espécies estudadas.

Trianoski et al. (2013b) avaliando a estabilidade dimensional de espécies tropicais de Pinus encontraram valores de 2,89, 6,47 e 10,22\% para contração nas direções radial, tangencial e volumétrica, respectivamente. Esses valores demonstram que os resultados para Pinus oocarpa obtidos nesse trabalho estão próximos aos encontrados na literatura. Tais propriedades são de extrema importância, uma vez que quanto maior o índice de anisotropia, maior a tendência de os painéis apresentarem empenamentos, com reflexo na estabilidade dimensional do material (IWAKIRI, 2005).

A norma NBR 7190 (ABNT, 1997) classifica, a madeira conforme seu coeficiente de anisotropia, variando entre 1,50 e superior a 2,0; sendo aquelas até 1,50 consideradas excelentes, de 1,50 a 2,0 normais, e acima de 2,0 consideradas

Tabela 1 - Valores médios de umidade (\%), densidade básica $\left(\mathrm{g} / \mathrm{cm}^{3}\right)$ e densidade aparente $\left(\mathrm{g} / \mathrm{cm}^{3}\right)$ para as madeiras estudadas. Table 1 - Mean values of moisture (\%), basic density $\left(\mathrm{g} / \mathrm{cm}^{3}\right)$ and apparent density $\left(\mathrm{g} / \mathrm{cm}^{3}\right)$ for the studied woods.

\begin{tabular}{cccc}
\hline Tratamentos & Umidade $(\%)$ & $\begin{array}{c}\text { Densidade Aparente } \\
\left(\mathrm{g} / \mathrm{cm}^{3}\right)\end{array}$ & $\begin{array}{c}\text { Densidade Básica } \\
\left(\mathrm{g} / \mathrm{cm}^{3}\right)\end{array}$ \\
\hline Acrocarpus fraxinifolius & $10,57^{(0,81)} \mathrm{A}$ & $0,58^{(0,02)} \mathrm{A}$ & $0,45^{(0,02)} \mathrm{A}$ \\
Pinus oocarpa & $10,69^{(0,11)} \mathrm{A}$ & $0,69^{(0,09)} \mathrm{A}$ & $0,47^{(0,02)} \mathrm{A}$ \\
Castilla ulei & $10,81^{(0,59)} \mathrm{A}$ & $0,58^{(0,01)} \mathrm{A}$ & $0,47^{(0,02)} \mathrm{A}$ \\
\hline
\end{tabular}

Médias seguidas pela mesma letra não diferem estatisticamente entre si pelo teste de Tukey a 95\% de significância. Valores entre parênteses se referem a desvio padrão.

Tabela 2 - Valores médios de contração na direção radial e tangencial, da variação volumétrica e do coeficiente de anisotropia para as madeiras estudadas.

Table 2 - Mean values of radial and tangential shrinkage, volumetric variation and anisotropy coefficient for the studied wood.

\begin{tabular}{ccccc}
\hline Tratamentos & Radial (\%) & Tangencial (\%) & Variação volumétrica & $\begin{array}{c}\text { Coeficiente de } \\
\text { anisotropia }\end{array}$ \\
\hline $\begin{array}{c}\text { Acrocarpus } \\
\text { fraxinifolius }\end{array}$ & $2,51^{(1,18)} \mathrm{A}$ & $6,69^{(1,28)} \mathrm{B}$ & $11,65^{(2,00)} \mathrm{B}$ & $2,67^{(0,48)} \mathrm{B}$ \\
Pinus oocarpa & $2,61^{(0,68)} \mathrm{A}$ & $4,29^{(1,32)} \mathrm{A}$ & $8,63^{(1,57)} \mathrm{A}$ & $1,64^{(0,25)} \mathrm{A}$ \\
Castilla ulei & $5,77^{(0,80)} \mathrm{B}$ & $9,33^{(0,61)} \mathrm{C}$ & $17,82^{(1,47)} \mathrm{C}$ & $1,62^{(0,27)} \mathrm{A}$ \\
\hline
\end{tabular}

Médias seguidas pela mesma letra não diferem estatisticamente entre si pelo teste de Tukey a 95\% de significância. Valores entre parênteses se referem a desvio padrão. 
ruins. Diante dos resultados obtidos, Castilla ulei e Pinus oocarpa podem ser consideradas normais, enquanto Acrocarpus fraxinifolius é considerada com qualidade ruim, podendo apresentar maiores propensões às rachaduras, sendo, consequentemente, menos estáveis.

Os resultados encontrados para a composição química das madeiras de Acrocarpus fraxinifolius, Pinus oocarpa e Castilla ulei estão apresentados na Tabela 3.

Ao avaliar os valores médios para os extrativos, todos foram estatisticamente diferentes. A madeira de Acrocarpus fraxinifolius apresentou o maior teor médio de extrativos (8,15\%), seguida da madeira de Castilla ulei (4,53\%) e, por fim, a madeira de Pinus oocarpa (4,03\%).

Fonte; Trianoski (2015) relatam que espécies com elevados teores de extrativos podem gerar painéis com deficiência na colagem. Esse fenômeno ocorre, provavelmente, porque os extrativos presentes no material lignocelulósico podem migrar para a superfície durante o processo de secagem e/ou prensagem da chapa e, consequentemente, inativar a superfície e dificultar a molhabilidade da madeira e penetração do adesivo (FRIHART; HUNT, 2010; BUFALINO et al., 2012a, 2012b).

Para lignina, as madeiras apresentaram valores estatisticamente iguais, 40,34, 31,21 e $26,08 \%$, respectivamente para Acrocarpus fraxinifolius, Castilla ulei e Pinus oocarpa. A lignina é um adesivo natural e, portanto, maiores teores são desejáveis para a produção de painéis de madeira reconstituída (KHEDARI et al., 2004; BUFALINO et al., 2012a). Dessa forma, espera-se que os compensados produzidos com madeiras com altos teores dessas substâncias apresentem maior resistência à água e melhores propriedades mecânicas (KHEDARI et al., 2004).

Para o teor de cinzas, todas as madeiras apresentaram valores médios estatisticamente diferentes. A madeira de Castilla ulei apresentou o maior valor médio (1,81\%), seguida da madeira de Acrocarpus fraxinifolius $(0,64 \%)$ e, por fim, a madeira de Pinus oocarpa (0,26\%). De acordo com Soares et al. (2017) altos teores de minerais podem resultar no bloqueio de grupos químicos reativos para a adesão com adesivos polares, afetando, assim, a qualidade da colagem e o desempenho mecânico das chapas reconstituídas. Com isso, observa-se que a madeira de Castilla ulei poderá apresentar problemas na colagem, devido à presença de mais de $1 \%$ de cinzas em sua constituição.

Na Tabela 4 pode-se observar os valores médios de MOE e MOR para o ensaio de flexão estática.

Quando avaliado o MOE e MOR, observa-se que a madeira de Pinus oocarpa apresentou maior valor (7844,57 e $125,86 \mathrm{MPa}$, respectivamente). Isso pode ser explicado pelas maiores densidades aparente e básica evidenciadas na espécie. Em ensaios realizados pelo Laboratório de Tecnologia da Madeira - LTM (2009), o módulo de ruptura para a madeira de Acrocarpus fraxinifolius foi de $77 \mathrm{MPa}$ e para o módulo de elasticidade foi de $9374 \mathrm{MPa}$.

Trianoski et al. (2014) trabalhando com madeiras de espécies de pinus tropicais obteve valores de MOE e MOR de

Tabela 3 - Composição química da madeira de Acrocarpus fraxinifolius, Pinus oocarpa e Castilla ulei.

Table 3 - Chemical composition of the wood of Acrocarpus fraxinifolius, Pinus oocarpa and Castilla ulei.

\begin{tabular}{ccccc}
\hline Material & Extrativos (\%) & Lignina (\%) & Holocelulose (\%) & Cinzas (\%) \\
\hline Acrocarpus & $8,15^{(0,10)} \mathrm{C}$ & $40,34^{(3,25)} \mathrm{C}$ & $50,88^{(3,33)} \mathrm{A}$ & $0,64^{(0,02)} \mathrm{B}$ \\
fraxinifolius & $4,03^{(0,17)} \mathrm{A}$ & $26,08^{(2,50)} \mathrm{A}$ & $69,63^{(2,63)} \mathrm{C}$ & $0,26^{(0,02)} \mathrm{A}$ \\
Pinus oocarpa & $4,53^{(0,26)} \mathrm{B}$ & $31,21^{(2,02)} \mathrm{B}$ & $62,45^{(5,06)} \mathrm{B}$ & $1,81^{(0,17)} \mathrm{C}$ \\
Castilla ulei & &
\end{tabular}

Médias seguidas pela mesma letra não diferem estatisticamente entre si pelo teste de Tukey a 95\% de significância. Valores entre parênteses se referem a desvio padrão. 
Tabela 4 - Valores médios de $\mathrm{MOE}(\mathrm{MPa})$ e $\mathrm{MOR}(\mathrm{MPa})$ para flexão estática.

Table 4 - Mean values of MOE (MPa) and MOR $(\mathrm{MPa})$ for static bending.

\begin{tabular}{ccc}
\hline Tratamentos & MOE $(\mathrm{MPa})$ & MOR $(\mathrm{MPa})$ \\
\hline Acrocarpus fraxinifolius & $2183,53^{(727,83)} \mathrm{A}$ & $51,84^{(12,50)} \mathrm{A}$ \\
Pinus oocarpa & $7844,57^{(2210,00)} \mathrm{B}$ & $125,86^{(20,28)} \mathrm{B}$ \\
Castilla ulei & $4333,1666^{(399,57)} \mathrm{A}$ & $84,91^{(4,60)} \mathrm{A}$ \\
\hline
\end{tabular}

Médias seguidas pela mesma letra não diferem estatisticamente entre si pelo teste de Tukey a 95\% de probabilidade. Valores entre parênteses se referem a desvio padrão.

7993 e $70 \mathrm{MPa}$, respectivamente. Notou-se que para a espécie de Pinus oocarpa o presente trabalho apresentou maior valor médio de módulo de ruptura. Foram obtidos valores médios para o MOE e o MOR da madeira de Castilla ulei de 8924 e 55,8 MPa, respectivamente (LPF/IBAMA) (IBAMA, 1997; IBDF, 1981, 1988; SOUZA et al., 1997).

De maneira geral, os valores médios de resistência mais elevados encontrados na espécie de Pinus oocarpa são explicados pela sua maior massa específica. Trianoski et al. (2014) afirmam que a massa específica influencia acentuadamente as propriedades mecânicas, pois está diretamente relacionada com o módulo de ruptura e módulo de elasticidade. Maiores valores de resistência mecânica das madeiras tendem a proporcionar melhores características aos painéis sarrafeados, melhorando a sua estabilidade dimensional e fornecendo propriedades mecânicas superiores.

\section{Propriedades físicas dos compensados}

$\mathrm{Na}$ Tabela 5 encontram-se os valores médios de umidade, densidade aparente e absorção de água dos painéis compensados sarrafeados das três espécies estudadas.
Para densidade aparente dos painéis nota-se que não houve diferença entre os tratamentos estudados. Os valores obtidos foram compatíveis aos encontrados pela ABIMCI (2007) para painéis comerciais produzidos no Brasil, compreendidos entre $0,476 \mathrm{~g} / \mathrm{cm}^{3}$ e $0,641 \mathrm{~g} / \mathrm{cm}^{3}$. Iwakiri et al. (2013) trabalhando com painéis compensados multilaminados obteve valores próximos aos obtidos neste trabalho, apresentando densidade aparente entre $0,394 \mathrm{~g} / \mathrm{cm}^{3}$ e $0,419 \mathrm{~g} / \mathrm{cm}^{3}$.

No teste de absorção de água, pode-se observar que ocorreu diferença significativa entre as médias de todos os tratamentos. O compensado sarrafeado produzido com a madeira de Pinus oocarpa apresentou os maiores valores $(67,77 \%)$. Isso pode ter ocorrido devido à madeira de Pinus apresentar maiores valores de holocelulose (Tabela 3). Essa fração carboidrato da madeira apresenta grupos hidroxílicos livres, principalmente na região amorfa da celulose e hemiceluloses, que são as principais responsáveis pela ligação com as moléculas de água (GUIMARAES JUNIOR et al., 2013).

Arruda et al. (2011) trabalhando com compensados mul tilaminados produzidos com lâminas de Schizolobium ama-

Tabela 5 - Valores médios de Umidade (\%), Densidade Aparente $\left(\mathrm{g} / \mathrm{cm}^{3}\right)$ e Absorção de Água (\%) dos painéis.

Table -5 - Mean values of Moisture (\%), Apparent Density $\left(\mathrm{g} / \mathrm{cm}^{3}\right)$ and Water Absorption (\%) of the panels.

\begin{tabular}{cccc}
\hline Tratamentos & Densidade Aparente $\left(\mathrm{g} / \mathrm{cm}^{3}\right)$ & Absorção de Água (\%) & Umidade $(\%)$ \\
\hline Acrocarpus & $0,54^{(0,06)} \mathrm{A}$ & $56,98^{(6,59)} \mathrm{B}$ & $12,62^{(0,50)} \mathrm{A}$ \\
fraxinifolius & $0,53^{(0,06)} \mathrm{A}$ & $67,77^{(12,93)} \mathrm{C}$ & $12,63^{(0,33)} \mathrm{A}$ \\
Pinus oocarpa & $0,57^{(0,02)} \mathrm{A}$ & $46,88^{(3,87)} \mathrm{A}$ & $12,80^{(0,53)} \mathrm{A}$ \\
Castilla ulei & &
\end{tabular}

Médias seguidas pela mesma letra não diferem estatisticamente entre si pelo teste de Tukey a 95\% de significância. Valores entre parênteses se referem a desvio padrão. 
zonicum obteve valores de absorção de água entre 75,04\% e $77,35 \%$, compatíveis aos observados neste trabalho.

Os valores médios de umidade ficaram próximos dos $12 \%$, não sendo diferentes estatisticamente, por consequência do período de climatização adequado, com temperatura e umidade controladas, pelo qual os painéis passaram. Os valores encontrados estão próximos ao observado por Campos et al. (2014) que obteve resultados próximos a 12\% na produção de compensados de pinus e eucalipto produzidos com adesivo PVA.

\section{Propriedades mecânicas dos compensados}

A Tabela 6 apresenta os valores médios da tensão máxima de cisalhamento na linha de cola para os painéis sarrafeados das três madeiras estudadas.

Tabela 6 - Valores médios de tensão máxima de cisalhamento $(\sigma)$ na linha de cola para os painéis sarrafeados. Table 6 - Maximum values of shear stress $(\sigma)$ in the glue line for the twined panels.

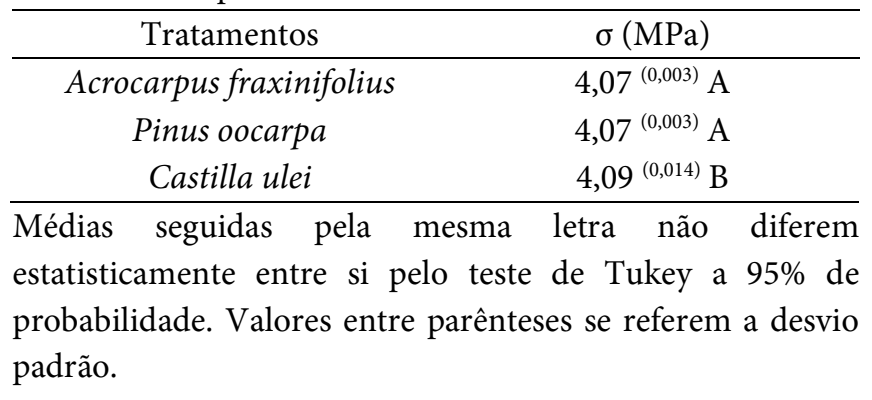

Para a tensão máxima de cisalhamento, os valores variaram entre 4,07 $\mathrm{MPa}$ e 4,09 $\mathrm{MPa}$, sendo o melhor desempenho observado para Castilla ulei. De acordo com a norma americana ASTM D-5751 (2005), o valor médio de resistência ao cisalhamento em linha de cola deve ser de pelo menos $60 \%$ do valor obtido para o ensaio da madeira sólida, que nesse caso corresponderia a 7,2; 3,5 e 5,4 MPa para Pinus oocarpa, Castilla ulei e Acrocarpus fraxinifolius, respectivamente. Nota-se que apenas Castilla ulei atendeu tal normativa. Contudo em trabalho realizado por Campos et al. (2014) com painéis compensados de Pinus sp. e Eucalyptus sp. colados com adesivo PVA, as médias obtidas foram de 3,57 e 3,76 MPa de tensão de cisalhamento para Eucalyptus sp. e Pinus sp., respectivamente. Tais valores apontam que os painéis sarrafeados produzidos neste trabalho encontram-se acima aos encontrados na literatura.

Na Tabela 7 podem-se observar os valores médios de MOE e MOR paralelo e perpendicular a direção das fibras dos sarrafos.

Observa-se na Tabela 7 que o MOE paralelo foi maior para o Pinus oocarpa, com valor médio de 7256,7 MPa. Já para Castilla ulei e Acrocarpus fraxinifolius os valores médios foram inferiores e não diferem estatisticamente entre si, com valores de 1955,7 $\mathrm{MPa}$ e 2019,2 $\mathrm{MPa}$, respectivamente. Os resultados estão próximos aos obtidos por Campos et al. (2014), em compensados de pinus e eucalipto produzidos com utilização de adesivo PVA, com valores paralelos de 9026,5 e 8535,6 MPa, para eucalipto e pinus, respectivamente.

Já para MOR paralelo, os valores médios encontrados foram maiores para Pinus oocarpa (60,3 MPa), seguido por Acrocarpus fraxinifolius (45,1 MPa) e Castilla ulei (42,7 MPa),

Tabela 7 - Valores médios de $\mathrm{MOE}(\mathrm{MPa})$ e $\mathrm{MOR}(\mathrm{MPa})$ paralelo e perpendicular para os tratamentos.

Table 7 - Mean values of MOE (MPa) and MOR $(\mathrm{MPa})$ were parallel and perpendicular to treatments.

\begin{tabular}{ccccc}
\hline Tratamentos & MOR // $(\mathrm{MPa})$ & $\mathrm{MOR} \perp(\mathrm{MPa})$ & $\mathrm{MOE} / /(\mathrm{MPa})$ & $\mathrm{MOE} \perp(\mathrm{MPa})$ \\
\hline Pinus oocarpa & $60,3^{(16,7)} \mathrm{A}$ & $13,3^{(2,8)} \mathrm{A}$ & $7256,7^{(903,5)} \mathrm{B}$ & $2696,5^{(688,0)} \mathrm{B}$ \\
Castilla ulei & $42,7^{(3,1)} \mathrm{A}$ & $10,3^{(3,5)} \mathrm{A}$ & $5368,7^{(916,0)} \mathrm{A}$ & $1955,7^{(512,27)} \mathrm{A}$ \\
Acrocarpus fraxinifolius & $45,1^{(9,5)} \mathrm{A}$ & $12,9^{(1,2)} \mathrm{A}$ & $4344,8^{(881,6)} \mathrm{A}$ & $2019,2^{(540,73)} \mathrm{A}$ \\
\hline
\end{tabular}

Médias seguidas pela mesma letra não diferem estatisticamente entre si pelo teste de Tukey a 95\% de probabilidade. Valores entre parênteses se referem a desvio padrão. 
próximos aos observados por Campos et al. (2014), obtendo resultados entre 60,4 e 67,5 $\mathrm{MPa}$, para eucalipto e pinus, respectivamente. Todos os tratamentos estão acima do valor mínimo exigido pela norma ASTM D5572 (1999), que é de 13,8 MPa. Esses resultados podem ser atribuídos a boa colagem dos sarrafos com o adesivo PVA, considerando os corpos de prova com cortes paralelos ao sentido das fibras.

Para MOE perpendicular observa-se que os compensados sarrafeados de Pinus oocarpa atingiram o maior valor $(2696,5$ $\mathrm{MPa}$ ), seguido por Acrocarpus fraxinifolius (2019,2 MPa) e Castilla ulei (1955,7 MPa). Os resultados encontrados ficaram abaixo aos obtidos por Lima; Pio (2007), que trabalhando com compensados de duas espécies arbóreas obteve resultados variando de 5918,9 a 7174,5 $\mathrm{MPa}$ e de 6484,6 a 8480,5 MPa para Copaifera duckei e Eperua oleifera, respectivamente.

Já para MOR perpendicular, os valores encontrados foram 13,3 MPa para Pinus oocarpa, seguido por Acrocarpus fraxinifolius (12,9 MPa) e Castilla ulei (10,3 MPa). Estes valores são menores do que valores obtidos por Lima; Pio (2007), com médias de 51,54 MPa a 60,95 MPa e de 49,12 a 64,53 MPa para corpos de prova com direção perpendicular as fibras para Copaifera duckei e Eperua oleifera, respectivamente. Os valores não atingiram o mínimo exigido pela norma ASTM D5572 (1999) de 13,8 MPa, ficando os compensados de Pinus oocarpa e Acrocarpus fraxinifolius bastante próximos a exigência normativa.

\section{Conclusões}

- Para as propriedades físicas das madeiras avaliadas, não houve diferença significativa. Porém a madeira de Pinus oocarpa apresentou os maiores valores de MOE e MOR para a espécie citada e consequentemente em maiores valores para as propriedades mecânicas dos compensados sarrafeados.

- Os materiais utilizados para produção dos painéis apresentaram características de densidade aparente e umidade adequadas para a produção de painéis compensados sarrafeados.

- Em relação ao ensaio de cisalhamento na linha de cola dos painéis sarrafeados, os painéis produzidos com Castilla ulei obtiveram o maior valor médio. Devido à baixa presença de extrativos da madeira, que quando em altos teores, tende a reduzir a qualidade da colagem.

- Para MOR perpendicular nenhum dos tratamentos obteve valores mínimos exigidos pela norma ASTM D5572 (1999) de 13,8 MPa.

\section{Referências}

ABIMCI - ASSOCIAÇÃO BRASILEIRA DA INDÚSTRIA DE MADEIRA PROCESSADA MECANICAMENTE. Painéis de compensado de pinus - Catálogo Técnico. Curitiba, 2007. 4 p.

AMERICAN SOCIETY FOR TESTING AND MATERIALS. ASTM 5572. Standard Specification for Adhesives Used for Finger Joints in Nonstructural Lumber Products. West Conshohocken, United States, 1999. 17 p.

AMERICAN SOCIETY FOR TESTING AND MATERIALS. ASTM D 143. Standard methods of testing small clear specimens of timber. Philadelphia, 1995. 31 p.

AMERICAN SOCIETY FOR TESTING AND MATERIALS. ASTM D-5751: Adhesives used for laminate joints in nonstructural lumber products. West Conshohocken, PA, 2005. 11p.

ARRUDA, L. M. et al. Estudo preliminar das propriedades de compensados produzidos com lâminas de Paricá (Schizolobium amazonicum Huber ex Ducke) modificadas termomecanicamente. Ciência da Madeira, Pelotas, v. 02, n. 01, p. 29-42, Maio de 2011.

ASSOCIAÇÃO BRASILEIRA DE NORMAS TÉCNICAS. NBR 11941. Determinação da Densidade Básica. Rio de Janeiro, 2003. 6 p. 
ASSOCIAÇÃO BRASILEIRA DE NORMAS TÉCNICAS NBR 13999. Papel, cartão, pastas celulósicas e madeira: determinação do resíduo (cinza) após a incineração a $525^{\circ} \mathrm{C}$. Rio de Janeiro, 2017. 5 p.

ASSOCIAÇÃO BRASILEIRA DE NORMAS TÉCNICAS. NBR 14853. Madeira: determinação do material solúvel em etanol-tolueno e em diclorometano e em acetona. Rio de Janeiro, 2010. 3 p.

ASSOCIAÇÃO BRASILEIRA DE NORMAS TÉCNICAS. NBR 7190. Projeto de estruturas de madeira. Rio de Janeiro, 1997. $107 \mathrm{p}$.

ASSOCIAÇÃO BRASILEIRA DE NORMAS TÉCNICAS. NBR 7989. Pasta celulósica e madeira: determinação de lignina insolúvel em ácido. Rio de Janeiro, 2010. 6 p.

ASSOCIAÇÃO BRASILEIRA DE NORMAS TÉCNICAS. NBR 9484. Compensado: Determinação do teor de umidade. Rio de Janeiro, 2011. 2 p.

ASSOCIAÇÃO BRASILEIRA DE NORMAS TÉCNICAS. NBR 9485. Compensado: Determinação do teor de umidade. Rio de Janeiro, 2011. 2 p.

ASSOCIAÇÃO BRASILEIRA DE NORMAS TÉCNICAS. NBR 9486. Compensado: Determinação do teor de umidade. Rio de Janeiro, 2011. 2 p.

ASSOCIAÇÃO BRASILEIRA DE NORMAS TÉCNICAS. NBR 9533. Compensado: Determinação da resistência à flexão estática. Rio de Janeiro, 2012. 5 p.

ASSOCIAÇÃO BRASILEIRA DE NORMAS TÉCNICAS. NBR ISO 12466-1. Madeira Compensada - Qualidade de colagem parte 1: métodos de ensaio. Rio de Janeiro, 1986.

BORTOLIN, T. A.; TRENTIN, A. C.; PERESIN, D.; SCHNEIDER, V. E. Estimativa da geração de resíduos florestais no Brasil. $3^{\circ}$ Congresso Internacional de Tecnologias para o Meio Ambiente. Bento Gonçalves. 2012.

BRAZ, R. L.; OLIVEIRA, J. T. S.; ROSADO, A. M.; VIDAURRE, G. B.; PAES, J. B.; FILHO, M. T.; LOIOLA, P. L. Caracterização anatômica, física e química da madeira de clones de Eucalyptus cultivados em áreas sujeitas à ação de ventos. Ciência da Madeira (Brazilian Journal of Wood Science). v. 5, n. 2, p.127-137, 2014.

BUFALINO, L.; ALBINO, V. C. S.; SÁ, V. A.; CORRÊA, A. R. R.; MENDES, L. M.; ALMEIDA, N. A. Particleboards made from Australian red cedar: processing variables and evaluation of mixed species. Journal of Tropical Forest Science, v. 24, n. 2, p. 162-172, 2012 b.

BUfALINO, L.; PROTÁSIO, T. P. P.; COUTO, A. M.; NASSUR, O. A. C.; SÁ, V. A.; TRUGILHO, P. F.; MENDES, L. M. Caracterização química e energética para aproveitamento da madeira de costaneira e desbaste de cedro australiano. Pesquisa Florestal Brasileira, v. 32, n. 70, p. 129 137, 2012a.

CAMPOS, C. I.; FERREIRA, B. S.; PRATES, G. A.; VALENTINA, L. V. O. D. Utilização de adesivo PVA em compensados de Pinus sp. e Eucalyptus sp. Brazilian Journal of Biosystems Engineering. v. 8, p. 59-64, 2014.

FONTE, A. P. N.; TRIANOSKI, R. Efeito da gramatura sobre a qualidade de colagem lateral da madeira de Tectona grandis. Revista de Ciências Agroveterinárias, Lages, v.14, n.3, p.224-233, 2015.

FRIHART, C. R.; HUNT, C. G. Adhesives with wood materials: bond formation and performance. In: FOREST PRODUCTS LABORATORY. Wood handbook: wood as an engineering material. Washington, DC: USDA, 2010. p. 10/1$10 / 24$.

GUIMARAES, I. L.; GUIMARÃES JÚNIOR, J. B.; LISBOA, F. J. N. ; ANDRADE, C. R. ; SIQUEIRA, H. F. Caracterização química e física da madeira de procêdencias de Eucalyptus. Enciclopédia Biosfera, v. 9, p. 636, 2013.

INSTITUTO BRASILEIRO DE DESENVOLVIMENTO FLORESTAL. Madeiras da Amazônia: características e utilização. Brasília, 1981. v. 1, 113 p.

INSTITUTO BRASILEIRO DE DESENVOLVIMENTO FLORESTAL. Madeiras da Amazônia: características e utilização. Brasília, 1988. v. 2, 236 p.

INSTITUTO BRASILEIRO DO MEIO AMBIENTE E DOS RECURSOS NATURAIS RENOVÁVEIS. Madeiras da Amazônia: características e utilização. Brasília, 1997. v. 3, 141 p.

IWAKIRI, S. Painéis de Madeira Reconstituída. Curitiba: Fundação de Pesquisas Florestais - FUPEF, Universidade Federal do Paraná, 2005.

IWAKIRI, S.; DA CUNHA, A. B.; TRIANOSKI, R.; BRAZ, R. L.; DE CASTRO, V. G.; KAZMIERCZAK, S.; PINHEIRO, E.; RANCATTI, H.; SANCHES, F. L. Produção de Painéis 
Compensados Fenólicos com Lâminas de Madeira de Sequoia sempervirens. Floresta e Ambiente, v. 20, n.2, p. 264-270, 2013.

IWAKIRI, S.; POTULSKI, D.; SANCHES, F. G.; TRIANOSKI, R. Avaliação do potencial de uso da madeira de Acrocarpus fraxinifolius, Grevilea robusta, Melia azedarach e Toona ciliata para produção de painéis OSB. Cerne, Lavras, v. 20, n. 2, p. 277-284, abr./jun. 2014.

KHEDARI, J.; NANKONGNAB, N.; HIRUNLABH, J.; TEEKASAP, S. New low-cost insulation particleboards from mixture of durian peel and coconut coir. Building and Environment, v. 39, n. 1, p. 59-65, 2004.

\section{LABORATÓRIO DE TECNOLOGIA DA MADEIRA. Banco} de dados do Laboratório de Tecnologia da Madeira. Curitiba: UFPR, 2009.

LIMA, E. G.; SILVA, D. A. Resíduos gerados em indústrias de móveis de madeira situadas no pólo moveleiro de ArapongasPR. Revista Floresta, v. 35, n.1, p.105-116, 2005.

LIMA, N. N.; PIO, N. S. Avaliação da gramatura de cola na propriedade de flexão estática em painéis compensados de Copaifera duckei Dwayer e Eperua oleifera Ducke. Acta Amazonica, Manaus, v. 37, n. 03, p. 347-352, 2007.

MATTOS, B. D. et al. Variação axial da densidade básica da madeira de três espécies de gimnospermas. Revista Brasileira de Ciências Agrárias, v. 6, n. 1, 2011, p 121-126.

MENDES, L.M.; SILVA, J.R.M.; TRUGILHO, P.F., LIMA, J.T. Variação da densidade da madeira de Pinus oocarpa Schiede ex Schltdl. no sentido longitudinal dos caules. Cerne, v.5, n.1, p. 105-111, 1999.

MINISTÉRIO DO MEIO AMBIENTE - MMA. Aproveitamento de resíduos e subprodutos florestais, alternativas tecnológicas e propostas de políticas ao uso de resíduos florestais para fins energéticos. Curitiba, PR. 2009.

SOARES, S. S.; GUIMARÃES JÚNIOR, J. B.; MENDES, L. M.; MENDES, R. F.; PROTÁSIO, T. P.; LISBOA, F. J. N. Valorização do bagaço de cana-de-açúcar na produção de painéis aglomerados de baixa densidade. Ciência da Madeira (Brazilian Journal of Wood Science). v.8, n.2, p. 64-73, 2017.

SOUZA, M. H. et al. Madeiras tropicais brasileiras. Brasília: IBAMA, 1997. 152 p.
STCP. Otimização da gestão de resíduos e o desenvolvimento florestal. II Encontro Nacional de Gestão de Resíduos ENEGER. Curitiba. 2011.

TRIANOSKI, R.; IWAKIRI, S.; MATOS, J. L. M.; PRATA, J. G. Propriedades físicas e mecânicas de painéis de madeira aglomerada de Acrocarpus fraxinifolius, compostos com diferentes percentuais de casca. Ciência Florestal [online], Santa Maria, v. 23, n. 4, p. 761-769, 2013.

TRIANOSKI, R.; MATOS, J. L. M.; IWAKIRI, S.; PRATA, J. G. Avaliação da estabilidade dimensional de espécies de pinus tropicais. Floresta Ambiente, Rio de Janeiro, v. 20, p. 398406, 2013.

TRIANOSKI, R.; MATOS, J. L. M.; IWAKIRI, S.; PRATA, J. G. Avaliação das propriedades mecânicas da madeira de espécies de Pinus tropicais. Scientia Forestalis, Piracicaba, v. 42, n. 101, p. 21-28, mar. 2014. 